\title{
Article
}

\section{Prevalence of diagnosis and treatment continuity predictors of new patients in a paediatric psychiatry clinic}

\author{
Ah Rah Lee ${ }^{1}$ and Geon Ho Bahn ${ }^{2, *}$ \\ 1 Graduate School of Medicine, Kyung Hee University, Seoul, Korea; preppie i@naver.com \\ 2 Department of Psychiatry, College of Medicine Kyung Hee University, Seoul, Korea; mompeian@khu.ac.kr \\ ${ }^{*}$ Correspondence: mompeian@khu.ac.kr
}

\begin{abstract}
This study analysed trends of first-time patients visiting the paediatric psychiatry clinic in a university hospital. The medical records from 2009 to 2016 of first-time patients visiting the Kyung Hee university hospital were reviewed, focusing on children in grades 1-12. We analysed the prevalence rate of psychiatric disorders per 100,000 general populations by gender and grade, and the characteristics of patients who sought outpatient care more than three times. The study included 1,467 participants, of which 931 were males $(63.5 \%)$. The number of male patients per 100,000 populations significantly decreased from 4.14 in 2009 to 2.03 in 2016 . While hyperkinetic disorders had the highest prevalence in males, neurotic disorders were most frequent in females. Prevalence of disruptive behaviour disorders in males and mental retardations in females decreased significantly during the study period. The factors affecting continuity were being female, studying in grades 7-12, and diagnosis of depressive, hyperkinetic, and tic disorders. Physicians should consider the new paediatric patients' gender, grade, and expected diagnosis from their first visit to improve treatment compliance.
\end{abstract}

Keywords: Treatment adherence; child; adolescent; trend; outpatient clinic; new patient

\section{Introduction}

There is a growing interest in mental health issues in children and adolescents [1]. In the U.S., 43,283 parents of children aged 3-17 years participated in a national survey, which revealed that $7.4 \%$ of the children had current behavioural/conduct problems, $7.1 \%$ had current anxiety problems, and 3.2\% had current depression [2]. The national survey of children's health in the U.S., which relies on parent reports, estimated that the prevalence of anxiety or depression among children aged 6 to 17 years rose from $5.4 \%$ in 2003 to $8.4 \%$ in 2011-2012 [3]. Based on a meta-analysis report summarising the prevalence of common mental disorders among adolescents aged between 10 and 19 years, the global prevalence of common mental health disorders - which was measured using the 12-item General Health Questionnaire with cut-off points of 4 and 3-were 25.0\% and $31.0 \%$, respectively [4]. Suicide is the leading cause of death among teenagers since 2009 in the Republic of Korea and is also of concern to Korean mental health experts [5]. Although there are very few suicides among elementary school students, they adversely impact the people around them, including their families [6].

Interestingly, the prevalence rate estimated through epidemiological investigations and the prevalence rate of diagnosis observed in clinical practice may be different. A child and adolescent mental health survey in Seoul reported the estimated prevalence of full-syndrome and subthreshold Diagnostic and Statistical Manual of Mental Disorders, Fourth Edition (DSM-IV) disorders as $16.2 \%$ and $28.1 \%$, respectively [7]. However, the diagnosis prevalence rate of psychiatric disorders was $1.95-2.38 \%$ based on the Korean Health Insurance Review and Assessment Service (HIRA) sample data for children aged 0-18 years during 2010-2015 [8]. These gaps can also be identified in individual diagnoses. While attention-deficit/hyperactivity disorder (ADHD) is reported among 5-8\% of 
children worldwide, the average annual diagnosis prevalence of ADHD in Korean children is $0.357 \%$ and exposure to anti-ADHD medication is $0.248 \%$ [9]. The rate of exposure to anti-ADHD medication in Taiwan was $0.34 \%$ [10] and $0.48 \%$ in Germany [11]. Though the prevalence of disruptive mood dysregulation disorder is estimated as $2-5 \%$ in DSM-5 [12], the weighted prevalence was $0.3-0.76 \%$ in Taiwanese children as observed in a national epidemiological study [13].

Interest in psychiatric disorders among children and adolescents has heightened; however, there is not enough data based on recent clinical practice. Several studies have reported limited data for specific years and conditions on clinical aspects of first-time paediatric patients, but few studies have identified changes and long-term trends for the entire population. This study seeks to bridge the gap in existing research by analysing the epidemiological trends for an eight-year period of new patients in the paediatric psychiatry outpatient clinic of a university hospital compared to the total population.

\section{Materials and methods}

\subsection{Participants}

This study included children from grades 1-12 who were first-time patients at the outpatient clinic of paediatric psychiatry, Kyung Hee University Hospital during an eight-year period from January 2009 to December 2016. Infants and pre-schoolers between the ages of $0-6$ years were excluded because they needed to be monitored for a certain period for an accurate diagnosis and were nevertheless difficult to diagnose definitely [8]. The study period was set to 2009-2016 because the hospital's electronic medical records system was changed before and after the study period, and there were no changes in the psychiatrists and clinical psychologists during that period, thereby ensuring consistency between the medical record system and the medical staff.

\subsection{Methods}

During the study period, gender, grade, and diagnosis were retrospectively analysed using medical records of first-time patients by year. To analyse the factors influencing treatment continuity, participants with less than three outpatient visits were classified as the early dropout group, while those with three or more outpatient visits were considered the treatment continuity group; this was based on the study on early dropout factors by Pelkonen et al. [14]. For clinical diagnosis, we used the Korean Standard Classification of Diseases (KCD) [15], which is based on the International Classification of Diseases [16].

The age groups were analysed using three-year intervals (grade 1-3, 4-6, 7-9, 10-12) based on a previous study reporting the diagnosis prevalence of psychiatric disorders in the $0-18$ years age group [8].

To compensate for the limitations of single hospital data, the diagnosis rate to the total population was calculated by reflecting the total population in the middle of the year [17]. In other words, diagnosis prevalence was analysed based on the total population aged 6-18 years per year.

The KCD codes for diagnosis were limited to psychiatric disorders ( $\mathrm{F}$ codes), symptoms and signs and abnormal clinical and laboratory findings, not elsewhere classified ( $R$ codes), and factors influencing health status and contact with health services ( $Z$ codes) (Table S1). The psychiatrists are often unable to make a psychiatric diagnosis during a child's first visit to the psychiatric clinic. Therefore, a tentative diagnosis was attached with an $\mathrm{R}$ or $\mathrm{Z}$ code based on the primary symptom. If the psychiatric diagnosis was determined during the treatment, an F code was attached. Once an F code diagnosis was made, the $R$ and $Z$ codes were not included in the analysis. In cases where the treatment was discontinued before the F code diagnosis was made, either the $\mathrm{R}$ or $\mathrm{Z}$ code diagnosis was included in the analysis.

In the case of psychiatric disorders, six higher diagnostic groups were established in the order of frequency based on HIRA sample data research [8] and the preliminary 
analysis of this data. Hyperkinetic disorders (F90) and tic disorders (F95) belonged to a single diagnostic code. The other four groups of diagnoses were classified together with similar disorders: neurotic disorders (F40-48), depressive disorders (F32, 33), disruptive behaviour disorders (F91-94), and mental retardations (F70-79). Disorders that affect a small number of patients were classified as 'others' which include the following diagnoses: psychotic disorders (F20-29), bipolar disorders (F30, 31), eating and sleep disorders (F50, 51), personality disorders (F60-65), psychological developmental problems (F80-84), and mental disorders, not otherwise specified (F99). The analysis of diagnosis per person allowed for redundancy.

\subsection{Statistical Analysis}

The changes in the diagnosis prevalence of psychiatric disorders from 2009 to 2016 by gender, grade, and year were examined according to the rate of increase or decrease in the annual average and the significance of the trends was estimated using a trend test. The prevalence rate was converted to a percentage of the F code diagnosed by psychiatrists per 100,000 population included in each group as designated by the total mid-year population.

To investigate the factors affecting treatment continuity, the patients were divided into groups based on the number of outpatient visits, and the most effective logistic regression model was selected through the stepwise method based on gender, grade, estimated diagnosis, and the number of diagnosis redundancy. A generalised linear model with the interaction term of year and grade was estimated to compare the annual growth rate of each grade by gender.

The statistical significance was set at 0.05 for all analyses, and the data were analysed in SAS 9.4 (Statistical Analysis System version 9.4, SAS Institute, Cary, NC, USA) using R 4.0.2 (https://cran.r-project.org/).

\section{Results}

Of the 1467 participants, 931 (63.5\%) were male and 536 (36.5\%) were female. Between 2009 and 2016, the total number of cases per 100,000 population of the age group (6-18 years) decreased significantly from 3.24 to 1.88 , and the number of male patients decreased significantly from 4.14 to 2.03 (Table 1). The number of female patients decreased from 2.24 to 1.72 , which was not statistically significant. The overall ratio of male and female patients decreased from 1.87 to 1.29 during the study period, which was not statistically significant.

In the overall distribution of participants by grade, the largest proportion of students were in grades 1-3. In terms of year-over-year changes, changes in grades 1-3 were higher than those for other grades in 2009, 2011, and 2013 and changes in grades 10-12 were higher than those for the others in 2010, 2012, and 2014-2016. The number of males decreased significantly in grades $1-3,7-9$, and 10-12. The number of females showed a significant decline in grades 4-6 and 7-9, and increased in grades 10-12 with no statistical significance (Figure 1). 
Table 1. Number of first-time patients according to the year, gender, and grades (per 10,000)

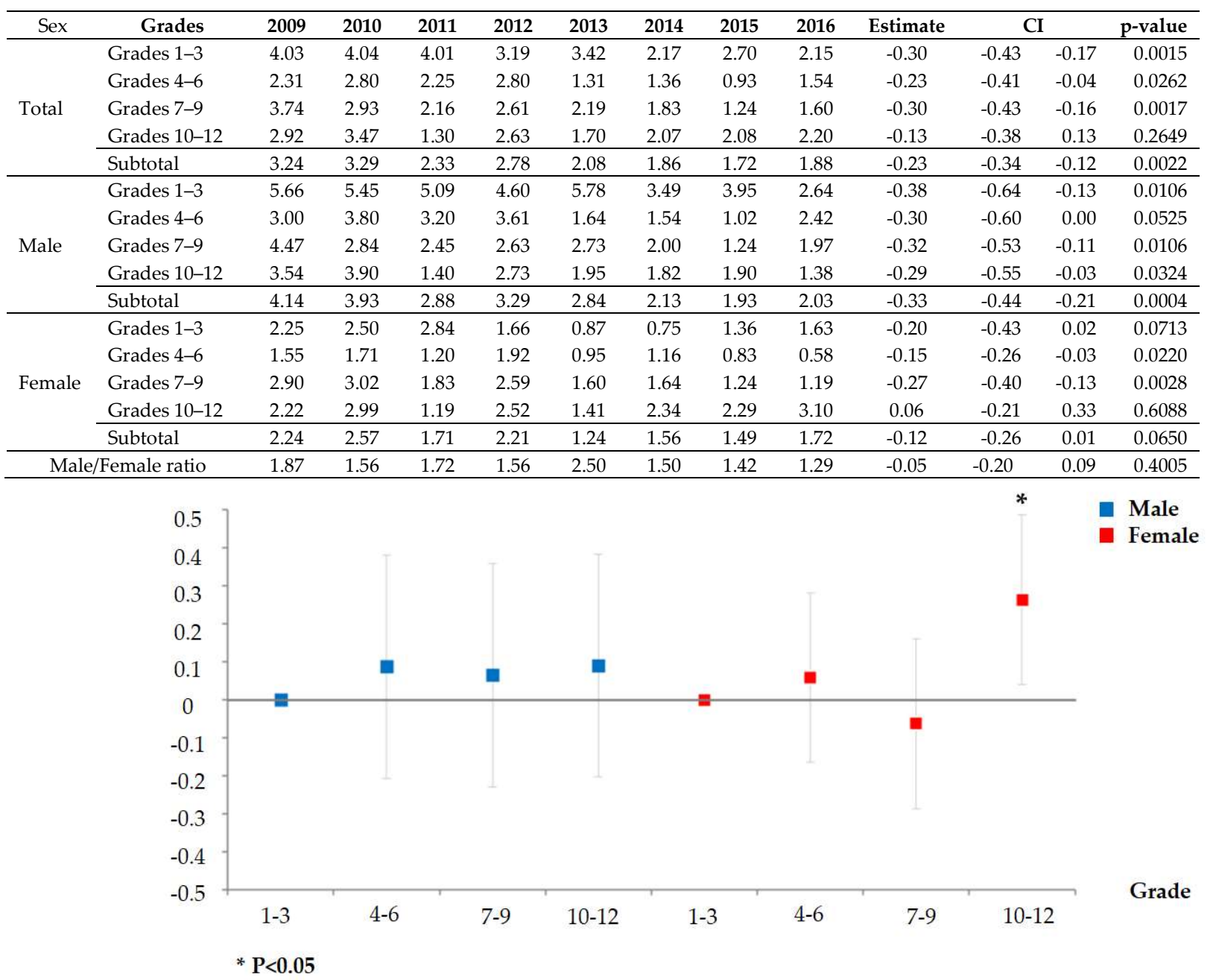

Figure 1. Distribution of new patients according to sex and grades (reference group: $1^{\text {st }}$ graders). 
In terms of the distribution of diagnostic codes, F90 was the highest, followed by F40-48, F95, others, F70-79, F32-33, and F91-94 (Figure 2). F90 had the highest diagnostic rate during the study period, but was on the decline. F91-94 diagnoses significantly decreased with year. In females, the diagnostic distribution was followed by F40-48, F90, F32-33, others, F70-79, F95, and F91-94 (Figure 3). F70-79 diagnoses significantly decreased with year. The most common single diagnosis in females was other anxiety disorders (F41), followed by depressive episodes (F32), somatoform disorders (F45), and F90.

The total number of $\mathrm{R}$ codes was 317 (males 62.8\%), and that of $\mathrm{Z}$ codes was 98 (males 68.4\%).

Figure 2. Distribution of psychiatric disorders in male students by year.

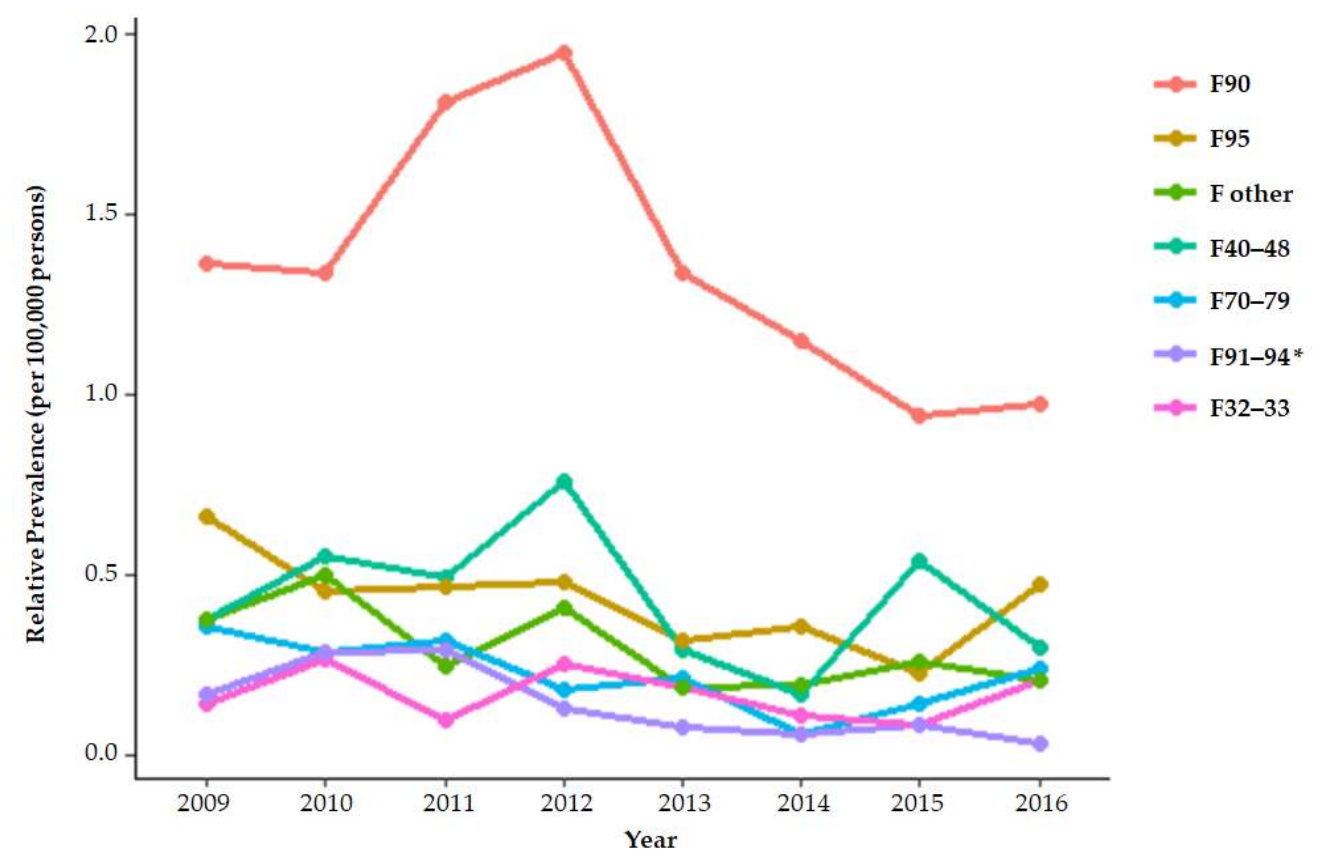

F32-33; Depressive disorder, F40-48; Personality disorder, F70-79; Mental and behavioural disorder, F90; Hyperkinetic disorders, F91-94; Disruptive behaviour disorder, F95; Tic disorders, Other; F07, F20-29, F30-31, F50-51, F60-69, F80, F81, F84, F99(F07; Personality and behavioural disorders due to brain disease, damage, and dysfunction, F20-29; Psychotic disorder, F30-31; Bipolar disorder, F50-51; Eating \& Sleep disorder, Neurotic disorder, F60-69; Personality disorder, F80; Learning disorder, F81; Specific developmental disorders of scholastic skills, F84; Pervasive developmental disorders, F99; Unspecified mental disorder F91-94, sloop: $-0.03(-0.06,-0.01)^{*}$ 


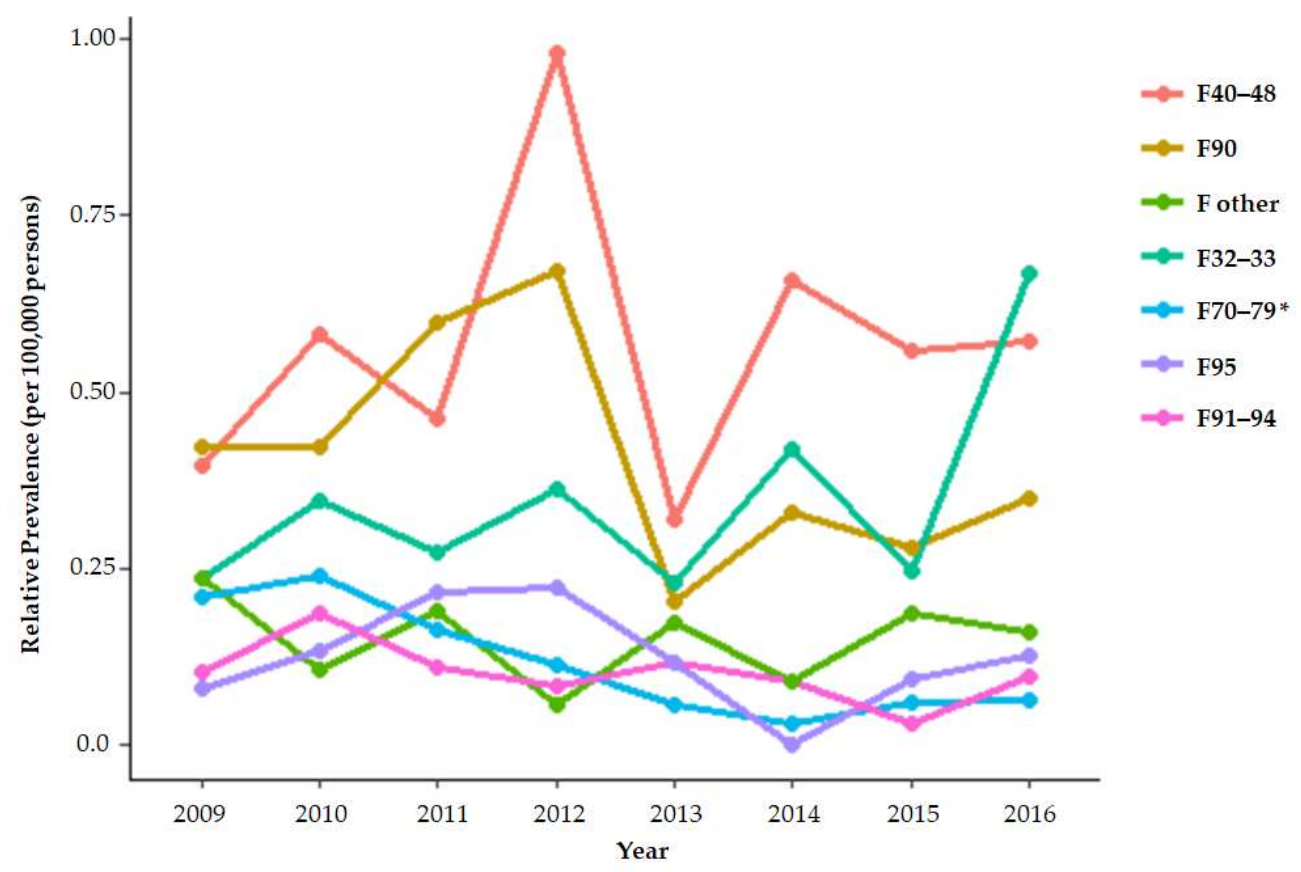

Figure 3. Distribution of psychiatric disorders in female students by year.

F32-33; Depressive disorder, F40-48; Personality disorder, F70-79; Mental and behavioural disorder, F90; Hyperkinetic disorders, F91-94; Disruptive behaviour disorder, F95; Tic disorders, Other; F07, F20-29, F30-31, F50-51, F60-69, F80, F81, F84, F99(F07; Personality and behavioural disorders due to brain disease, damage, and dysfunction, F20-29; Psychotic disorder, F30-31; Bipolar disorder, F50-51; Eating \& Sleep disorder, Neurotic disorder, F60-69; Personality disorder, F80; Learning disorder, F81; Specific developmental disorders of scholastic skills, F84; Pervasive developmental disorders, F99; Unspecified mental disorder F7079, sloop: $-0.03(-0.04,-0.01)^{*}$

Of the 1,467 participants, 645 (45.0\%) (423 males and 222 females) dropped out early (Table S3). The factors correlated with continuity of follow-up were patients who were female, in grades 7-12, with psychiatric disorders including F32-33, F90, and F95, and with one or more psychiatric diagnosis (Table 2). 
Table 2. Factor analysis for the treatment continuity defined as more than three outpatient visits

\begin{tabular}{lllrrrr}
\hline Item & & OR & & CI & & p-value* \\
\hline \multirow{2}{*}{ Sex } & Male & 1.00 &. &. & \\
& & Female & 1.36 & 1.07 & 1.74 & 0.0125 \\
\hline \multirow{2}{*}{ Grades } & Grades 1-3 & 1.00 & $\cdot$ & $\cdot$ & $\cdot$ \\
& & Grades 4-6 & 1.04 & 0.75 & 1.44 & 0.8261 \\
& & Grades 7-9 & 1.39 & 1.01 & 1.90 & 0.0422 \\
& & Grades 10-12 & 1.81 & 1.30 & 2.52 & 0.0005 \\
\hline \multirow{2}{*}{ Diagnosis } & Depressive episode, Recurrent & & 1.65 & 1.08 & 2.51 & 0.0198 \\
\cline { 2 - 7 } & depressive disorder (F32-33) & & 3.14 & 2.32 & 4.26 & $<.0001$ \\
\cline { 2 - 7 } & Hyperkinetic disorder (F90) & & 2.83 & 1.89 & 4.24 & $<.0001$ \\
\cline { 2 - 7 } & Tic disorder (F95) & 0 & 1.00 & & & \\
\hline \multirow{2}{*}{ Number of Psychiatric Diagnosis\# } & 1 & 2.02 & 1.44 & 2.82 & $<.0001$ \\
& & $\geq 2$ & 1.63 & 1.02 & 2.61 & 0.0416 \\
\hline
\end{tabular}

${ }^{*}$ Multiple logistic regression test

\#Number of psychiatric diagnosis was focused on F32-33, F40-48, F70-79, F90, F91-94, and F95.

\section{Discussion}

In this study, a significant decline in the total number of first-time patients and male patients is observed, even if the statistics are corrected to reflect the decline in the birth rate in the Republic of Korea. These results are consistent with decreased prevalence of diagnosis of psychiatric disorders in children and adolescents under 19 years of age in HIRA statistics [8]. Those trends are contrary to the increase in the prevalence of psychiatric disorders in children and adolescents in screening-oriented epidemiological studies [18]. While it may vary depending on the target disorders or research design, there is a clear gap between epidemiological prevalence and treatment rate, with only $25-35 \%$ of affected children and adolescents accessing treatment [19]. These differences suggest that there may be differences in estimated prevalence and diagnosis prevalence on clinical practice, depending on epidemiological investigation methods or national healthcare systems.

Gender ratio of psychiatric disorders changes during the transition from childhood to adulthood because of biological and environmental factors. Based on 2011 HIRA data, in a study of the population under the age of 19 , the ratio of male to female patients with psychiatric disorders was 1.68:1 [20]. This study also showed that the percentage of male patients was higher than that of female patients. The sample data from the HIRA of 1,375,842 people in 2011 revealed that the male-to-female ratio for those aged 19-30 years was 48.1 to 51.9 [21]. A cross-national meta-analysis of mental disorders in the World Health Organisation World Mental Health Surveys also revealed that the prevalence of mental disorders is 1.1 times higher in women than in men [22]. What is noteworthy here is the cause of this reversal of the gender ratio. One of the reasons for this significant narrowing of gender differences was related to depressive disorders and the variation of gender role traditionality [22].

In this study, the number of male patients from grades 1 to 12 and female patients from grades 1 to 9 has decreased. Interestingly, the number of female patients in grades 10-12 has increased over the year, however, this increase was not statistically significant. Two factors could have contributed to this trend: first, the Adolescent Personality and Mental Health Screening Questionnaire, conducted annually for the $1^{\text {st }}, 4^{\text {th }}, 7^{\text {th }}$, and $10^{\text {th }}$ graders across the whole country in the Republic of Korea since 2012 and the increased number of treatment-linked cases; second, parents' awareness of youth mental disorders through media or public education [1]. In Kim's study [23], the student number of grades 1-3 was more than any other grades from 2004 to 2009, and that of grades 10-12 was more than any other grades from 2010 to 2013. During that period, the incidence of de- 
pressive disorders increased around grades 7 to 12 . One of the reasons why the grades 13 group is larger than other grades in the psychiatric outpatient clinic may be because the group has a higher diagnosis and treatment rate of ADHD patients [8]. From three population-based cohorts and a meta-analysis, it is evident that children and adolescents who are relatively younger than their classmates have a higher risk of receiving an ADHD diagnosis [24].

The most common diagnosis in female patients in this study was F41, followed by F32, F45, and F90. The lifetime diagnosis of anxiety or depression among children aged 6 to 17 years increased based on the parent report in the US [3]. While current anxiety increased significantly, current depression did not change. In a study on the prevalence of paediatric mental disorders in Taiwan, the lifetime prevalence was the highest for anxiety disorder, followed by ADHD, sleep disorder, and tic disorder [25]. Concurrent comorbidity and homotypic and heterotypic continuity from age 9 through 16 years were more marked in girls than in boys, and girls had a higher incidence of depressive disorders as they grew [26].

The most common diagnosis in male students in this study was F90. According to the Centre for Disease Control and Prevention in the U.S., ADHD was the most frequent psychiatric disorder among children under the age of 18 [27]. ADHD was the most common mental disorder in the population under the age of 19 based on the HIRA sample data, followed by other anxiety disorders (F41), depressive episodes (F32), somatoform disorders (F45), reaction to severe stress, and adjustment disorders (F43) [20]. A long-term comparison over a 26-year period (1980-2005) from a tertiary care centre for child and adolescent psychiatric service in India revealed the increase in registration with affective illnesses in the ages 10-15, which is reflective of a worldwide trend towards an earlier onset and increased prevalence of affective illnesses [28]. In a study of the prevalence rate for adolescents across three consecutive years for overall psychiatric disorders in Taiwan, the rates for ADHD and phobia decreased and the rates for major depression and substance use disorders increased [29]. Conduct disorder and ADHD were more prevalent among boys, and major depression and phobia were higher among girls.

In this study, a significant decrease in F91-94 was observed among male patients over the years, while F70-79 showed a decreasing trend in female patients. A comparison of the clinical profiles of patients in child psychiatry in Bahrain between 1981-1982 and 2011-2012 showed that the prevalence rate of conduct disorders and anxiety disorders were lower [30]. The reason for this drop in prevalence is more likely to be due to other reasons than due to a real decrease, such as in those diagnosed with ADHD or learning disorders instead of conduct disorders. In this study, if conduct disorder and ADHD coexist in the same patient, they may have been diagnosed with ADHD. The prevalence of conduct disorders also declined between 1998 and 2013-2014 among younger males according to a study in Australia, primarily due to a decline in the prevalence among males living in two-parent families [31]. A significant reduction in F70-79 may be due to early detection and early intervention among individuals with mental retardation during their preschool period compared to the past, resulting in improved adaptability [20]. However, the reason for the significant decline in females alone in this study should be identified in future studies.

From this study, significant factors for treatment continuity were female students, higher grades, one or more psychiatric diagnosis, and disorders such as depressive disorders, hyperkinetic disorders, and tic disorders. One of the causes that affect the duration of treatment among those in higher grades and in females may be associated with increased anxiety and depressive disorders in the concerned gender and age group [8]. A study based on inpatient adolescents found that being female was a predictor of change in global functioning [32]. Pelkonen et al. [14] also reported that not having a mood disorder and not using psychotropic medication were associated with early dropout of ad- 
olescents from outpatient psychiatric treatment. The younger the patients, the more likely they were to drop out early without commencement of pharmacotherapy in ADHD [33]. Younger children are at higher risk of receiving suboptimal care with psychotropic medications [34], and their medication adherence may reduce depending on their parents' adherence [35]. These results suggest that the factors of treatment compliance may vary depending on the target disorders, culture, age, and gender of the subjects, or the research design.

This study has several limitations. First, as the participants of this study are outpatients at one university hospital, one should be careful while applying the findings of this study to the entire general population. However, since it is based on medical records of the same medical staff in a single institution, consistency in care can be an advantage. Second, this study is a retrospective review of medical records and does not apply the planned diagnostic tools as in prospective studies. The fact that the same clinical psychologists conducted psychological evaluations also provides the advantage of increasing accuracy and consistency in diagnosis. Despite these limitations, this is the first study in Korea wherein the trends in outpatients have been verified by reflecting changes in the overall population. The identification of treatment continuity predictors in first-time patients is also the strength of this study.

\section{Conclusion}

While the number of first-time patients in a university hospital's paediatric psychiatry department significantly declined over eight years, the number of older female students did not decrease. Furthermore, while hyperkinetic disorder was commonly found in male students, anxiety and depressive disorders were observed in females. The analysis of treatment continuity factors suggests that being female, in a higher grade, and having several psychiatric disorders are important predictors.

\section{Supplementary Materials:}

Table S1. Diagnoses with F, R, and Z codes.

\begin{tabular}{|c|c|c|c|}
\hline \multicolumn{2}{|c|}{ Groups } & KCD-7 codes & Diagnosis \\
\hline \multirow{2}{*}{\multicolumn{2}{|c|}{ Depressive symptom (F32-33) }} & F32 & Depressive episode \\
\hline & & F33 & Recurrent depressive disorder \\
\hline \multirow{7}{*}{\multicolumn{2}{|c|}{ Neurotic symptom (F40-48) }} & F40 & Phobic anxiety disorders \\
\hline & & F41 & Other anxiety disorders \\
\hline & & $\mathrm{F} 42$ & Obsessive-compulsive disorder \\
\hline & & F43 & Reaction to severe stress, and adjustment disorders \\
\hline & & F44 & Dissociative [conversion] disorders \\
\hline & & F45 & Somatoform disorders \\
\hline & & F48 & Other neurotic disorders \\
\hline \multicolumn{2}{|c|}{ Low intelligence (F70-79) } & F70-79 & Mental retardation \\
\hline \multicolumn{2}{|c|}{ Hyperkinetic problems (F90) } & F90 & Hyperkinetic disorders \\
\hline \multirow{4}{*}{\multicolumn{2}{|c|}{ Disruptive behaviors (F91-94) }} & F91 & Conduct disorders \\
\hline & & F92 & Mixed disorders of conduct and emotions, \\
\hline & & F93 & Emotional disorders with onset specific to childhood, \\
\hline & & F94 & $\begin{array}{l}\text { Disorders of social functioning with onset specific to child- } \\
\text { hood and adolescence }\end{array}$ \\
\hline \multicolumn{2}{|c|}{ Tic symptoms (F95) } & F95 & Tic disorders \\
\hline \multirow[t]{3}{*}{ Others } & $\begin{array}{l}\text { Psychotic symptoms } \\
\text { (F20-29) }\end{array}$ & F20-29 & Schizophrenia, schizotypal and delusional disorders \\
\hline & Mood-bipolarity (F30- & F30 & Manic episode \\
\hline & & F31 & Bipolar affective disorder \\
\hline
\end{tabular}




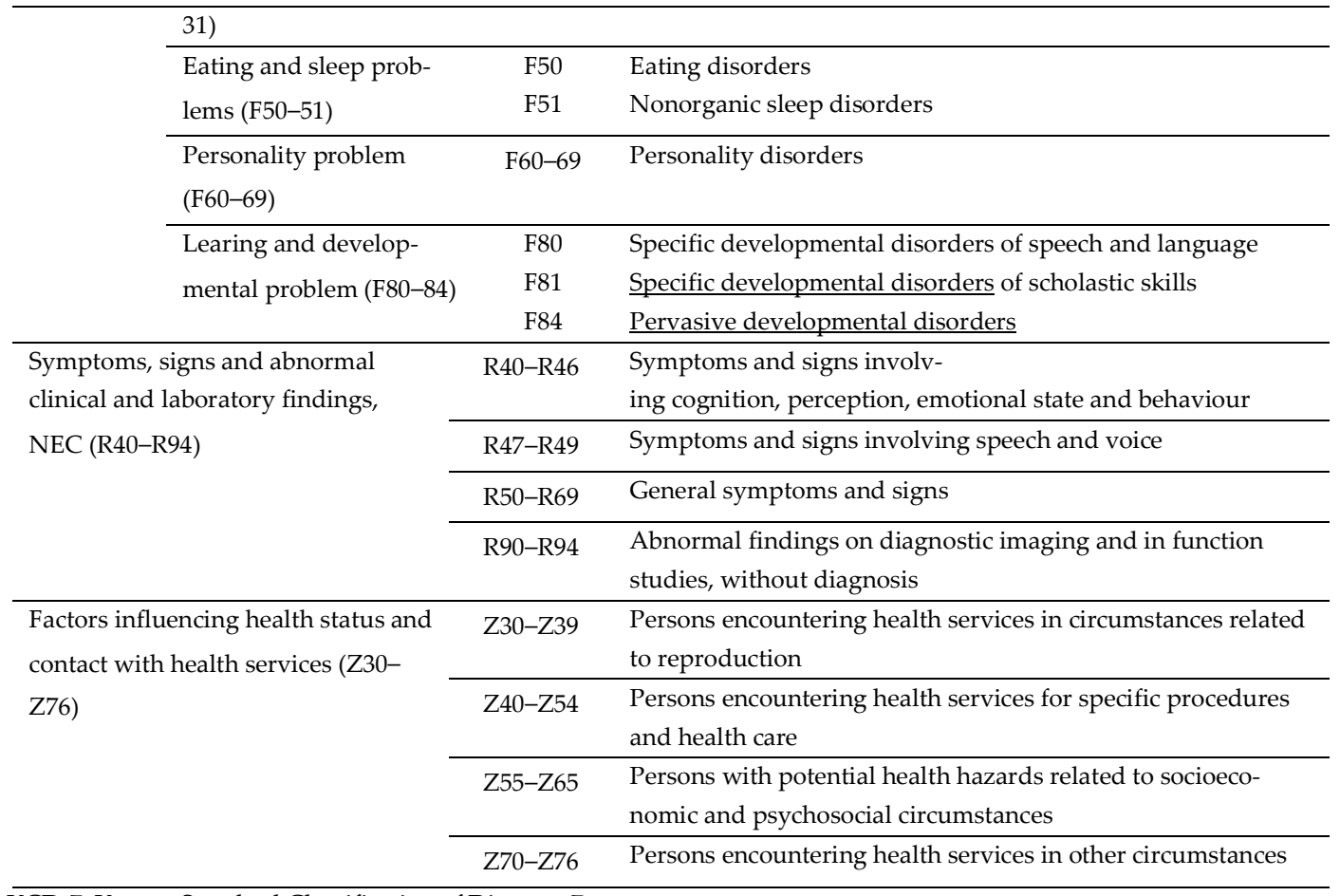

KCD-7: Korean Standard Classification of Diseases-7

Table S2. Number of first-time patients with psychiatric diagnosis by year.

\begin{tabular}{lrrrrrrrrrrrr}
\hline Diagnosis & $\mathbf{2 0 0 9}$ & $\mathbf{2 0 1 0}$ & $\mathbf{2 0 1 1}$ & $\mathbf{2 0 1 2}$ & $\mathbf{2 0 1 3}$ & $\mathbf{2 0 1 4}$ & $\mathbf{2 0 1 5}$ & $\mathbf{2 0 1 6}$ & estimate & CI & p-value \\
\hline F32-33 & 15 & 24 & 14 & 23 & 15 & 18 & 11 & 28 & 0.014 & -0.020 & 0.047 & 0.3655 \\
F40-48 & 31 & 45 & 37 & 65 & 22 & 28 & 37 & 28 & -0.007 & -0.077 & 0.063 & 0.8214 \\
F70-79 & 23 & 21 & 19 & 11 & 10 & 3 & 7 & 10 & -0.028 & -0.048 & -0.007 & 0.0155 \\
F90 & 74 & 72 & 96 & 101 & 58 & 53 & 42 & 44 & -0.061 & -0.147 & 0.025 & 0.1355 \\
F91-94 & 11 & 19 & 16 & 8 & 7 & 5 & 4 & 4 & -0.022 & -0.039 & -0.005 & 0.0187 \\
F95 & 31 & 24 & 27 & 27 & 16 & 13 & 11 & 20 & -0.022 & -0.048 & 0.004 & 0.0805 \\
Other & 25 & 25 & 17 & 18 & 13 & 10 & 15 & 12 & -0.019 & -0.035 & -0.004 & 0.0220 \\
\hline
\end{tabular}

F32-33; Depressive disorder, F40-48; Neurotic disorder, F70-79; Mental retardation, F90;Hyperkinetic disorders, F91-94; Disruptive behavior disorder, F95; Tic disorders, Other; (F20-29; Psychotic disorder, F30-31; Bipolar disorder, F50-51; Eating \& Sleep disorder, Neurotic disorder, F60-69; Personality disorder, F80-89; Disorders of psychological development, F99; Unspecified mental disorder)

Table S3. Clinical characteristics of early dropout group and treatment continuity group

by gender and grade.(\%)

\begin{tabular}{|c|c|c|c|c|c|c|c|c|c|}
\hline \multirow{2}{*}{\multicolumn{2}{|c|}{ Early dropout group }} & \multicolumn{2}{|c|}{ Total $(n=1432)$} & \multicolumn{2}{|c|}{ Male (n=911) } & \multicolumn{2}{|c|}{ Female $(n=521)$} & \multirow{2}{*}{$\begin{array}{l}\text { p-value } \\
<0.0017 \\
\end{array}$} & \multirow[t]{2}{*}{ p-value* } \\
\hline & & 645 & $(45.0)$ & 423 & $(46.4)$ & 222 & $(42.6)$ & & \\
\hline \multirow[t]{4}{*}{ Grade } & $1-3$ & 191 & $(29.6)$ & 143 & $(33.8)$ & 48 & $(21.6)$ & & \multirow{9}{*}{$<0.0001$} \\
\hline & $4-6$ & 136 & (21.1) & 94 & $(22.2)$ & 42 & (18.9) & & \\
\hline & 7-9 & 167 & (25.9) & 98 & $(23.2)$ & 69 & (31.1) & & \\
\hline & $10-12$ & 151 & $(23.4)$ & 88 & $(20.8)$ & 63 & $(28.4)$ & & \\
\hline \multicolumn{2}{|c|}{ Treatment continuity group } & 787 & $(55.0)$ & 488 & $(53.6)$ & 299 & $(57.4)$ & $<.0001$ & \\
\hline \multirow[t]{4}{*}{ Grade } & $1-3$ & 214 & $(27.2)$ & 156 & (32.0) & 58 & $(19.4)$ & & \\
\hline & $4-6$ & 142 & $(18.0)$ & 98 & (20.1) & 44 & (14.7) & & \\
\hline & $7-9$ & 202 & (25.7) & 118 & $(24.2)$ & 84 & $(28.1)$ & & \\
\hline & $10-12$ & 229 & $(29.1)$ & 116 & $(23.8)$ & 113 & $(37.8)$ & & \\
\hline
\end{tabular}

*chi-square test

${ }^{* *}$ Cochran-Mantel-Haenszeltest 


\begin{abstract}
Author Contributions:
Conceptualisation, G.H.B.; methodology, G.H.B. and A.R.L.; software, G.H.B.; formal analysis, G.H.B; investigation, G.H.B.; resources, G.H.B.; data curation, A.R.L.; writing-original draft preparation, G.H.B.; writing - review and editing, G.H.B. and A.R.L.; visualisation, A.R.L.; supervision, G.H.B.; project administration, G.H.B. All authors have read and agreed to the published version of the manuscript.
\end{abstract}

\title{
Funding:
}

This research received no external funding.

\section{Institutional Review Board Statement:}

The study was conducted according to the guidelines of the Declaration of Helsinki and was approved by the Institutional Review Board of Kyung Hee University Hospital (KMC IRB 2021-02-013-003).

\section{Informed Consent Statement:}

Patient consent was waived because this study was a retrospective medical record review of the patients.

\section{Data Availability Statement:}

Not applicable.

\section{Acknowledgments:}

Authors sincerely thank Soo Jin Chung of the statistics support office who enthusiastically helped us analyse the data in this study. Additionally, we would like to thank Editage (www.editage.co.kr) for English language editing.

\section{Conflicts of Interest:}

The authors declare no conflict interest.

\section{References}

1. Song, J.; Kweon, Y.S.; Hong, S.H.; Kim, J.; Chun, K.H.; Bahn, G.H.; Yook, K.; Shin, D.W.; Hong, H.J. Characteristics of first visit pediatric patients with suicidal ideation and behavior: an 8-year retrospective chart review. J Korean Acad Child Adolesc Psychiatr 2020, 31, 185-192.

2. Ghandour, R.M.; Sherman, L.J.; Vladutiu, C.J.; Ali, M.M; Lynch, S.E.; Bitsko, R.H.; Blumberg, S.J. Prevalence and treatment of depression, anxiety, and conduct problems in U.S. children. J Pediatr. 2019 206, 256-267.e3.

3. Bitsko, R.H.; Holbrook, J.R.; Ghandour, R.M.; Blumberg, S.J.; Visser, S.N.; Perou, R.; Walkup, J. Epidemiology and impact of healthcare provider diagnosed anxiety and depression among US children. J Dev Behav Pediatr 2018 39, $395-403$.

4. Silva, S.A.; Silva, S.U.; Ronca, D.B.; Gonçalves, V.S.S.; Dutra, E.S.; Carvalho, K.M.B. Common mental disorders prevalence in adolescents: a systematic review and meta-analyses. PLoS ONE 2020, 15, e0232007.

5. Kim, J.; Hong, S.H.; Hong, H.J. The impact of referral to mental health services on suicide death risk in adolescent suicide survivors. J Korean Acad Child Adolesc Psychiatr 2020, 31,177-184.

6. Hong, M.; Cho, H.N.; Kim, A.R.; Hong, H.J.; Kweon, Y.S. Suicidal deaths in elementary school students in Korea. Child Adolesc Psychiatr Ment Health 2017, 11, 53.

7. Park, S.; Kim, B.N.; Cho, S.C.; Kim, J.W.; Shin, M.S.; Yoo, H.J. Prevalence, correlates, and comorbidities of DSM-IV psychiatric disorders in children in Seoul, Korea. Asia Pac J Public Health 2015, 27, NP1942-1951.

8. Lee, S.Y.; Bahn, G.H. Patterns of the diagnosis prevalence of psychiatric disorders in the population aged 0-18 years based on the nationwide insurance sample data. J Korean Acad Child Adolesc Psychiatr 2020, 31, 214-224. 
9. Hong, M.; Kwack, Y.S.; Joung, Y.; Lee, S.I.; Kim, B.S.; Sohn, S.H.; Chung, U.; Yang, J.; Bhang, S.Y.; Hwang, J.; Choi, H.; Oh, I.H.; Lee, Y.J.; Bahn, G.H. Nationwide rate of attention-deficit hyperactivity disorder diagnosis and pharmacotherapy in Korea in 2008-2011. Asia-Pacific Psychiatry 2014, 6, 379-385.

10. Chien, I.C.; Lin, C.H.; Chou, Y.J.; Chou, P. (Prevalence, incidence, and stimulant use of attention-deficit hyperactivity disorder in Taiwan, 1996-2005: a national population-based study. Soc Psychiatry Psychiatr Epidemiol. 2012, 47, 1885-1890.

11. Kraut, A.A.; Langner, I.; Lindemann, C.; Banaschewski, T.; Petermann, U.; Petermann, F.; Mikolajczyk, R.T.; Garbe, E. Comorbidities in ADHD children treated with methylphenidate: a database study. BMC Psychiatry 2013, 13, 11.

12. American Psychiatric Association. Diagnostic and statistical manual of mental disorders, 5th edition: DSM-5; American Psychiatric Publishing: Arlington, VA, USA 2013.

13. Lin, Y.; Tseng, W.; Gau, S. Psychiatric comorbidity and social adjustment difficulties in children with disruptive mood dysregulation disorder: a national epidemiological study. J Affect Disord 2021; 281, 485-492.

14. Pelkonen, M.; Marttunen, M.; Laippala, P.; Lönnqvist, J. Factors associated with early dropout from adolescent psychiatric outpatient treatment. J Am Acad Child Adolesc Psychiatr 2000, 39, 329-336.

15. KOICD. Korean Standard Classification of Diseases, KCD-7, 2016:

https://www.koicd.kr/kcd/guide.do (Accessed on 1 April 2021)

16. World Health Organization. The ICD-10 classification of mental and behavioural disorders: clinical descriptions and diagnostic guidelines. World Health Organization: Geneva:, 1992.

17. Statistics Korea. Population in the middle of the year:

https://kosis.kr/statHtml/statHtml.do?orgId=101\&tblId=DT_1B040M1\&conn_path=I2 (accessed on )

18. Polanczyk, G.V.; Salum, G.A.; Sugaya, L.S.; Caye, A.; Rohde, L.A.; Annual Research Review: a meta-analysis of the worldwide prevalence of mental disorders in children and adolescents. J Child Psychol Psychiatr 2015, 56, 345-365

19. O’Brien, D.; Harvey, K.; Howse, J.; Reardon, T.; Creswell, C. Barriers to managing child and adolescent mental health problems: a systematic review of primary care practitioners' perceptions. Br J Gen Pract 2016, 66, e693-707.

20. Hwangbo, R.; Chang, H.; Hong, M.; Cho, S.; Bahn, G.H. The diagnostic distribution of psychiatric disorders among the population under 19 years Old: based on the national insurance data. J Korean Acad Child Adolesc Psychiatr 2016, 27, 139-145

21. Hwangbo, R.; Chang, H.; Bahn, G.H. Diagnostic distribution of psychiatric disorders among Korean young adults. J Korean Acad Child Adolesc Psychiatr 2020, 31, 89-87.

22. Seedat, S.; Scott, K.M.; Angermeyer, M.C.; Berglund, P.; Bromet, E.J.; Brugha, T.S.; Demyttenaere, K.;

de Girolamo, G.; Haro, J.M.; Jin, R.; et al. Cross-national associations between gender and mental disorders in the World Health Organisation World Mental Health Surveys. Arch Gen Psychiatr 2009, 66, 785-795.

23. Kim, H.; Jung, S.; Jung, C. The distributional changes in the first-visit psychiatric child and adolescent outpatients at a university hospital over a ten-year period. J Korean Acad Child Adolesc Psychiatr 2015, 26, 165-175

24. Caye, A.; Petresco, S.; Barros, A.J.D.; Bressan, R.A.; Gagelha, A.; Gonçalves, H.; Manfro, A.G.; Matijasevich, A.; Menezes, A.M.B.; Miguel, E.C.; et al. Relative age and attention-deficit/hyperactivity disorder: data from three epidemiological cohorts and a meta-analysis. J Am Acad Child Adolesc Psychiatr 2020, 59, 990-997.

25. Chen, Y.L.; Chen, W.J.; Lin, K.C.; Shen, L.J.; Gau, S.S. Prevalence of DSM-5 mental disorders in a nationally representative sample of children in Taiwan: methodology and main findings. Epidemiol Psychiatr Sci 2019, 29, e15. 
26. Costello, E.J.; Mustillo, S.; Erkanli, A.; Keeler, G.; Angold, A. Prevalence and development of psychiatric disorder in childhood and adolescence. Arch Gen Psychiatr 2003, 60, 837-844.

27. Perou, M.; Rebecca, H.; Stephen, J.; Pastor, Pa.; Reem, M.; Joseph, C.; Sarra, L.; Alex, E.; Susanna, N.; Laura, A.; Sharyn, E.; Jeffery, E.; Brody, D.; Catherine M.; William, W.; Baio, J.; Avenevoli, S.; Michael, D.; Larke, N. Centers for Disease Control and Prevention (CDC) Mental health surveillance among children-United States, 2005-2011. MMWR Suppl. 2013, 62, 1-35.

28. Malhotra, S.; Biswas, P.; Sharan, P.; Grover, S. Characteristics of patients visiting the child \& adolescent psychiatric clinic: a 26-year study from north India. J Indian Assoc Child Adolesc Ment Health 2007, 3, 53-60.

29. Gau, S.; Chong, M.Y.; Chen, T.H.H.; Cheng, A.T.A. A 3-year panel study of mental disorders among adolescents in Taiwan. Am J Psychiatr 2005, 162, 1344-1350.

30. Al-Ansari, A.M. Characteristics of child and adolescent populations visiting a public child and adolescent psychiatric clinic in Bahrain: A 30-year comparative analysis. The Arab Journal of Psychiatry 2015, 25, 32-38.

31. Sawyer, M.G.; Reece, C.E.; Sawyer, A.C.P.; Johnson, S.E.; Lawrence, D. Has the prevalence of child and adolescent mental disorders in Australia changed between 1998 and 2013 to 2014? J Am Acad Child Adolesc Psychiatr 2018, 57, $343-350$.

32. Kennedy, J.; Hembry, P.; Green, D.; Skuse, D.; Lewis, S. Predictors of change in global psychiatric functioning at an inpatient adolescent psychiatric unit: a decade of experience. Clin Child Psychol Psychiatr 2020, 25, 471-482.

33. Hong, M.; Lee, W.H.; Moon, D.S.; Lee, S.M.; Chung, U.; Bahn, G.H. A 36 month naturalistic retrospective study of clinic-treated youth with attention-deficit/hyperactivity disorder. J Child Adolesc Psychopharmacol 2014, 24, 341-346.

34. Dinnissen, M.; Dietrich, A.; van der Molen, J.H.; Verhallen, A.M.; Buiteveld, Y.; Jongejan, S.; Troost, P.W.; Buitelaar, J.K.; Hoekstra, P.J.; van den Hoofdakker, B.J. Prescribing antipsychotics in child and adolescent psychiatry: guideline adherence. Eur Child Adolesc Psychiatr 2020, 29, 1717-1727.

35. Nagae, M.; Nakane, H.; Honda, S.; Ozawa, H.; Hanada, H. Factors affecting medication adherence in children receiving outpatient pharmacotherapy and parental adherence. J Child Adolesc Psychiatr Nurs 2015, 28, 109-117. 